\title{
Culture et information. Le Centre culturel suédois et sa création
}

Andreas Hellenes

Université d'Oslo - Institut d'Études Politiques de Paris

Dans cet article, nous allons traiter de la création du Centre culturel suédois à Paris (Svenska kulturhuset $i$ Paris), qui a ouvert ses portes au public pour la première fois en janvier I97I, et qu'on appelle aujourd'hui l'Institut suédois à Paris ${ }^{\mathrm{T}}$. Les sources les plus importantes dans la réalisation de ce projet sont les archives inédites de l'Institut. Nous allons aborder le sujet par une contextualisation des relations franco-suédoises, puis parler du projet du Centre culturel suédois plus spécifiquement et examiner ses activités pour enfin esquisser le nouveau champ de recherche qui s'ouvre, au croisement entre histoire culturelle et histoire politique, à travers l'étude d'un tel établissement.

D'abord, afin de contextualiser brièvement cet épisode dans l'histoire des relations culturelles franco-suédoises, précisons qu'il s'agit d'une période qui représente un tournant, à la fois dans le contexte suédois et le contexte français. Ce tournant marque une réorientation vers ce que l'on a appelé la politique étrangère active, den aktiva utrikespolitiken, en Suède (Östberg \& Andersson, 20I3 : 276-277). Une critique sévère de l'action américaine au Vietnam constitue l'exemple le plus proéminent d'une série d'interventions très médiatisées de la part du nouveau premier ministre Olof Palme pendant ses premiers mandats, de 1969 en I976. La politique de neutralité qui avait caractérisé l'immédiat après-guerre s'est transformée, notamment sous l'action de Palme lui-même, en un engagement actif sur le plan international en faveur des mouvements de décolonisation au tiers-monde, un soutien à l'ANC en Afrique du Sud et une condamnation des dictatures militaires du Sud de l'Europe. C'est à la même époque que l'image de la

How to cite this book chapter:

Hellenes, A. 20 I 5 . Culture et information. Le Centre culturel suédois et sa création. In: Cedergren, M. et Briens, S. (eds.) Médiations interculturelles entre la France et la Suède. Trajectoires et circulations de I945 à nos jours. Pp. 99-I Io. Stockholm: Stockholm University Press. DOI: http://dx.doi.org/Io.I6993/bad.h. License: CC-BY 
Suède comme le pays progressiste par excellence a atteint son apogée à l'échelle internationale, et notamment en France. Jean-Jacques ServanSchreiber est le premier à avoir employé le terme de "modèle suédois" dans son livre de 1967 , Le défi américain, où il a consacré un chapitre à la Suède comme alternative au modèle américain ou japonais pour la France contemporaine (Servan-Schreiber, I967 : 312). Deux ans plus tard, la Suède a servi de point de référence dans la campagne présidentielle. Les candidats Alain Poher et Gaston Deferre ont fait écho au fondateur de l'Express. Le candidat gaulliste Georges Pompidou, quant à lui, a évoqué la possibilité d'instaurer en France un socialisme à la suédoise, mais avec «plus de soleil » (Faramond, 2000:39). Le Nouvel Observateur a peu de temps après les élections remarqué qu'en France, " la Suède est à la mode. Non pas en tant que destination touristique, mais en tant que vogue politique " (Martinet, I969). Et en effet, il nous semble possible de conclure qu'en France, dans un climat politique caractérisé par le lendemain du tumultueux Mai 68 et le départ du général de Gaulle, la Suède est parvenue à une position de référence politique dans le débat public - bien sûr, il faut le préciser, aux côtés d'autres régimes : il suffit d'évoquer par exemple la référence chinoise pour les groupes d'extrême gauche.

À Stockholm, les acteurs politiques et médiatiques ont rapidement remarqué cet intérêt international et notamment français pour le « modèle suédois ${ }^{3}$ ". Au cours de la même période, à savoir à la fin des années I960, l'Institut suédois, l'organisme chargé de promouvoir la Suède à l'étranger, a été réorganisé (Glover, 20I2 : I37-I38). Le Parlement a modifié son statut, d'institution semi-privée financée par l'État et les secteurs d'exportation, en une fondation publique à but non lucratif, financée entièrement par le Ministère des affaires étrangères. C'était durant cette période de réforme que le Centre culturel suédois à Paris a été conçu et créé. Le projet représente un pas radical dans l'histoire de la promotion culturelle suédoise à l'étranger. Jusque-là, aucun des bureaux étrangers - il y en avait eu à Paris, à Londres et à Prague - n'avait été un institut culturel à proprement parler ${ }^{4}$. Tous les acteurs impliqués soulignent le caractère unique du projet parisien 5 .

Ce contexte, ou peut-être mieux, ces contextes, rendent la création du Centre culturel suédois intéressante. Une question essentielle est, bien entendu, celle de savoir pourquoi le Ministre des finances suédois Gunnar Sträng, réputé pour être parcimonieux, a mis de l'argent sur la table pour acquérir un vieux palais à Paris. Dans la partie suivante, nous allons pourtant nous intéresser à un autre aspect de 
l'histoire, à savoir celle du projet d'un Centre culturel suédois à Paris, et plus précisément aux significations du terme "culturel ", et aux différentes notions de «culture ». Ce n'est pas seulement une question de vocabulaire, puisque la notion même de culture a donné lieu à un institut assez différent du projet qui était proposé par les acteurs initiaux.

\section{Les variétés d'instituts culturels}

L'historien grec Gregory Paschalidis a dans un article tracé l'évolution historique des centres culturels étrangers comme institution, depuis leur premier déploiement par les grandes puissances européennes au XIXe siècle jusqu'à leur adoption après la guerre froide par un grand nombre de pays. Selon lui :

Les différentes phases du développement de cet instrument politique démontrent sa versatilité unique et son adaptabilité à une variété de contextes et de fonctions, ainsi qu'à, au niveau plus général, son rôle stratégique dans le travail et les processus de la politique culturelle extérieure ${ }^{6}$. (Paschalidis, 2009: 275)

L'exemple du Centre culturel suédois est intéressant à cet égard. Avec sa création, deux institutions culturelles suédoises à Paris ont été réunies sous le même toit : le bureau parisien de l'Institut suédois, Svenska institutet, et l'Institut Tessin. Ouverts respectivement en I946 et en I933, ces instituts ont incarné deux visions très différentes des relations culturelles. L'Institut Tessin a été conçu comme un musée et une bibliothèque d'art suédois. Il a été fondé et dirigé par l'historien de l'art Gunnar W. Lundberg, conseiller culturel à l'Ambassade de Suède à Paris, avec le soutien des cercles académiques suédois, particulièrement dans le domaine des lettres et de l'histoire de l'art. L'Institut Tessin avait, à partir de 1963 , un statut comparable à celui des instituts suédois de Rome et d'Athènes, des centres de recherche consacrés à l'archéologie et aux études classiques, mais sans avoir les mêmes ressources. Dans le contexte de ces instituts, l'historien suédois Frederick Whitling a parlé de diplomatie académique, afin de saisir heuristiquement le mélange de diplomatie politique et culturelle effectué par des universitaires dans des fonctions semi-diplomatiques en tant que directeurs des instituts culturels ou des académies, entre la culture nationale et l'internationalisme culturel (Whitling, 20 I I : 645-668). Quand l'Institut Tessin au début des années I960 a été obligé de quitter ses 
locaux de la rue de Tournon, Lundberg et la Société Tessin ont réussi à convaincre l'État suédois d'acheter de nouveaux bâtiments à Paris en I965, à savoir l'Hôtel de Marle, au prix d'un million de couronnes. Une condition de la vente était la rénovation complète de l'hôtel; cette transaction a de fait été effectuée trois ans après l'introduction de la loi Malraux sur la préservation du patrimoine historique ${ }^{7}$. Au cours d'une période de cinq ans, de 1965 à I970, toutes les constructions parasites rajoutées au palais au fil du temps furent démolies et les bâtiments originaux rénovés, sous la supervision des autorités patrimoniales françaises. Le coût total du projet s'est élevé à huit millions de couronnes ${ }^{8}$.

Le rêve de Lundberg était d'y créer un institut de caractère muséal, dont le travail serait consacré aux relations artistiques et culturelles entre les deux pays, où les universités suédoises pourraient proposer à ses étudiants des cours spécialisés en histoire de l'art et où les artistes et chercheurs suédois venant à Paris pourraient être logés ; le tout dans une combinaison de musée et de bibliothèque d'art ${ }^{9}$. Lundberg se voyait lui-même comme le directeur naturel d'un tel établissement.

L'initiative du projet, bien qu'émanant de Lundberg, ne relevait cependant pas de ses compétences. Suivant une ligne d'activité différente, le bureau parisien de l'Institut suédois était impliqué dans une série d'activités d'information (ce que l'on appelait en suédois Upplysningsverksambet, ou par la suite Sverige-information) visant le public français, telles que la distribution de brochures, le contact entre les milieux universitaires français et suédois, et la promotion de la Suède contemporaine en France. Il organisait également des voyages d'études et des programmes de bourses pour les Français désireux d'aller en Suède. Nous regroupons ici, à l'instar de l'historien anglais Nicholas Cull, ces activités dans le concept de diplomatie publique: «La diplomatie publique est la tentative d'un acteur international de conduire une politique étrangère en engageant des publics étrangers ${ }^{\mathrm{I0}}$, (Cull, 2008 : xv). Le bureau parisien de l'Institut suédois était donc responsable de ce que l'on pourrait appeler les activités de diplomatie publique suédoise en France.

\section{Politiques culturelles et notions changeantes de culture}

La période suivant l'achat de l'Hôtel de Marle par l'État suédois en I965 et l'ouverture du Centre culturel fournit de nombreux exemples d'un nouveau champ politique émergent, à savoir celui des politiques 
culturelles. Le projet du Centre culturel était en effet dirigé par le Département des affaires culturelles du Ministère de l'Éducation du jeune Olof Palme ${ }^{\mathrm{II}}$. Ce dernier avait initié le travail qui allait aboutir à la proposition de I974 d'une nouvelle politique culturelle, I974 års Kulturpolitik, développée justement par le Département ${ }^{\mathrm{I2}}$. Cette section était également d'une importance vitale en ce qui concernait le projet d'un centre culturel suédois à Paris. Ancien ami de la France et de sa culture, Carl Johan Kleberg était responsable du projet au sein du ministère. Il est intéressant de noter que l'argument économique était complètement absent dans les enquêtes préliminaires sur le Centre culturel suédois. Alors que les enquêtes contemporaines sur l'avenir de l'Institut suédois ont traité en détail des effets économiques positifs des activités d'information, le Centre culturel, considéré comme une partie de la politique culturelle suédoise, était épargné par la demande de légitimer son existence au regard de son utilité économique.

Et nous nous retrouvons bien évidemment dans une période où les notions mêmes de culture subissaient de grandes transformations, en Suède comme ailleurs en Occident. Nous nous contenterons ici de citer un historien comme Anders Frenander ${ }^{13}$. Si l'on fait la comparaison entre les organes consultatifs du processus d'achat de l'Hôtel de Marle en I965 avec ceux de l'enquête du Ministère de l'Éducation de I970, l'extension du projet est révélatrice de ces évolutions. Un tournant vers le « culturel » dans un sens large, vers le monde culturel et ses acteurs, est très visible. Les Départements des langues romanes des universités d'Uppsala, de Lund et de Göteborg sont remplacés par les organisations et institutions centrales de la vie culturelle suédoise. Le but du Ministère était de donner la parole aux kulturarbetare, aux " ouvriers de la culture ", plus qu'aux universitaires. Le nouveau Centre culturel suédois à Paris allait ainsi devenir un centre pour tous les ouvriers culturels suédois.

La coordination et la centralisation organisationnelles étaient à l'ordre du jour : la standardisation et l'homogénéisation du matériel promotionnel et/ou d'information suédois. Au sein de l'Institut suédois, au Ministère des affaires étrangères comme au Ministère de l'Éducation, on était conscient de l'intérêt porté par la Suède contemporaine en France. Une réflexion sur la nature de l'image de la Suède, ou des images de la Suède, avait été mise en place au sein de l'Institut suédois déjà en 1956 (Hellenes, 2015) ${ }^{14}$. En I969, avec l'ouverture de la Maison de Suède (Sverigehuset) en plein cœur de Stockholm ce débat occupe la presse nationale. Incarnant parfaitement la volonté coordinatrice de 
certains décideurs suédois, l'immeuble dessiné par Sven Markelius pour la Maison de Suède regroupait les principaux acteurs de la diplomatie publique suédoise : l'Institut suédois, le Département de la presse du Ministère des affaires étrangers et l'Office du tourisme étant les premiers. Pour son ouverture, le Ministère a commissionné une exposition du jeune artiste Pär Stolpe, intitulée «Images de la Suède dans le monde » (Sverigebilder i världen). Cette exposition d'inauguration, bien qu'elle ait créé un scandale et ait été arrêtée après une seule journée, car considérée trop subversive, révèle néanmoins l'importance considérable que la direction de l'Institut suédois a donnée à l'image du pays (Glover, 2OII : I46-I 54).

Ce que nous tenons à souligner ici, c'est précisément qu'au fondement de ce processus il y a à la fois une idée très différente de ce que signifie le terme culture, et par conséquent aussi ce qu'allait être la nature d'un centre culturel suédois à l'étranger, et de la relation entre culture et information. Les plans initiaux du directeur Lundberg et de la Société Tessin étaient de leur côté très orientés vers les beauxarts, vers ce que l'on appelle en suédois högkultur's. À l'opposé, le plan de l'Institut suédois pour le nouveau centre culturel était celui d'un centre qui représentait la vie culturelle suédoise dans tous ses aspects, sélectionnés en coopération étroite avec les organisations représentatives du secteur culturel du pays et en combinaison avec des activités d'information promouvant la société suédoise auprès d'un public français et international ${ }^{16}$. L'Institut pensait faire ce choix en accord avec les demandes du public parisien. Ces idées correspondaient bien à la vision du Ministère de l'Éducation. Le directeur PerAxel Hildeman, dans un mémorandum envoyé aux représentants du monde artistique et culturel suédois, a proposé l'image du workshop pour le nouveau centre culturel, dans le sens " terrain de jeu et forum de débat ${ }^{17}$ ». Il a ensuite introduit l'idée de paquets thématiques comme colonne vertébrale des activités du centre. "Actualité et variation " étaient pour lui des mots-clés, et on devrait consciemment éviter tout effort de caractère muséal ${ }^{18}$. Hildeman, qui ne voyait pas de distinction nette entre les deux, avait toujours soutenu l'idée d'une combinaison de travail d'information et d'exchange culturel dans le contexte de l'Institut suédois ${ }^{19}$. Par conséquent, le Centre culturel suédois à Paris devrait unir les deux, à travers le principe de ne jamais organiser d'événement singulier, c'est-à-dire de toujours accompagner les manifestations culturelles de campagnes d'information sur la Suède contemporaine et vice versa. Ceci était d'autant plus vrai 
qu'un institut culturel suédois n'aurait jamais pu s'imaginer entrer en compétition en tant que musée avec les galeries, musées et autres institutions culturelles parisiennes sans égal au niveau international. C'était donc un plan d'action nettement différent de celui de l'Institut Tessin, et c'était la piste choisie par le Ministère de l'Éducation avant l'ouverture du Centre ${ }^{20}$.

\section{Les activités du Centre culturel suédois}

Passons maintenant, pour conclure cet article, brièvement aux activités menées par le nouveau Centre culturel durant ses premières années d'existence. On peut trouver un bon exemple de la stratégie de Hildeman dans une de ses premières manifestations, à savoir l'exposition "Évolution et aménagement du paysage en Suède » (Kulturlandskap, vad är det för nått). Cette exposition a été ouverte avec une réception qui également a servi à lancer un colloque de trois jours sur la politique environnementale, organisé par l'Ambassade de Suède, l'Institut suédois et les autorités environnementales française et suédoise. Le but principal du colloque était " en partie d'établir une coopération plus étroite entre les experts français et suédois de conservation environnementale, et en partie de créer de l'intérêt médiatique sur les questions d'environnement avant le grand colloque de l'ONU sur l'environnement humain, à être organisé à Stockholm en $1972^{21}$ ». En conclusion de l'exposition, L'Institut a organisé un débat public avec deux experts français, Christian Garnier et Jean-Baptiste Barde, les auteurs d'un volume sur la politique environnementale suédoise qui venait d'être publié. Leur ouvrage, L'Environnement sans frontières, faisait partie d'une collection publiée par la maison d'édition Seghers, "La Suède en question ", qui était financée par l'Institut suédois ${ }^{22}$.

L'exposition "Évolution et aménagement du paysage en Suède », sa circulation, son traitement par la presse française ainsi que son imbrication dans certains réseaux franco-suédois, pourrait faire objet d'un examen en détail. La même chose est vraie pour d'autres manifestations organisées au Centre culturel suédois au cours de ces premières années, où l'intérêt français pour la société et la politique suédoise, bref « le modèle suédois " était considéré comme un point fort par l'Institut suédois. D'autres colloques ont été organisés, par exemple sur l'égalité entre hommes et femmes en Suède, la politique culturelle et la démocratie sur le lieu de travail, où experts et politiques se sont réunis pour croiser expériences françaises et suédoises, et ont souvent 
été accompagnés de nouvelles expositions d'art ou des films thématiques. Voilà pourquoi nous aimerions, en conclusion, faire écho à l'historien suédois Andreas Åkerlund, qui, dans une anthologie consacrée aux relations culturelles entre la Suède et l'Allemagne, suggère que les instituts culturels pourraient constituer un point de départ très fructueux pour la recherche de transferts culturels (Åkerlund, 20I I : 5-I6). Ceci vaut certainement pour le Centre culturel suédois à Paris. L'examen du milieu autour du Centre constitue un bon point de départ pour une analyse détaillée des circulations d'individus, d'œuvres et de textes entre les sphères culturelles françaises et suédoises ; celleci conduit à s'interroger par exemple sur la nature des images de la Suède mobilisées en France suite à l'ouverture du Centre et sur le rôle des réseaux et d'individus français ou suédois dans ce que l'on pourrait appeler la communication de la Suède à Paris. Il est ainsi question de la rencontre d'une politique publique de promotion culturelle de la Suède à l'étranger et d'un contexte français et parisien dans lequel ces activités ont été formées, exprimées et envisagées pour un public plus ou moins spécifique.

\section{Conclusion}

Que peut-on dire, pour résumer, sur la création du Centre culturel suédois de Paris ? Justement que le Ministère de l'Éducation, plus que simplement offrir de nouveaux locaux à Gunnar W. Lundberg et l'Institut Tessin, a vu dans l'achat de l'Hôtel de Marle la possibilité de renforcer la présence culturelle suédoise dans la capitale française, à travers la création d'une institution jusqu'ici unique dans l'histoire culturelle suédoise, qui était également façonnée en fonction de ce que l'on considérait comme intéressant pour un public parisien. Contre la volonté de Lundberg, forcé de prendre sa retraite, l'Institut Tessin a été intégré dans le nouveau centre culturel, administré par l'Institut suédois, désormais une fondation publique consacrée avant tout à la représentation et la promotion de la culture et la société suédoises contemporaines ; autrement dit, un tournant de la diplomatie académique à la diplomatie publique. Pour conclure, nous aimerions suggérer que l'histoire de la création de l'Institut suédois nous montre que cette institution, plutôt que de faire partie d'une stratégie explicite de la part du Ministère des affaires étrangères, représente la victoire d'une notion de culture et des relations culturelles sur une autre. 


\section{Notes}

I. La création du Centre culturel suédois n'a jusqu'ici jamais fait l'objet d'analyses historiques. Elle a cependant été brièvement présentée par l'ancien directeur de l'Institut suédois, Per-Axel Hildeman, dans sa chronique des cinquante ans de l'Institut (Hildeman, I995:63), ainsi que par l'ancien directeur du Centre culturel suédois, Carl Henrik Svenstedt (Battail, I993 : 473-492).

2. D'après l'historien suédois Bo Stråth, c'est la première fois que le terme de «modèle suédois" est utilisé dans le discours politique (Stråth, 2006 : 39I).

3. Voir SIUP I968 : 5-6, SIUP i969: 45-46. Le Ministère des affaires étrangères commence dès I 968 la compilation de rapports annuels intitulée " $\mathrm{La}$ Suède dans la presse étrangère " (Sverige i utländsk press, SIUP).

4. Alors que le bureau à Prague ferma en 1948, le bureau londonien resta en vigueur jusqu'à son inclusion dans l'Ambassade en I973. Ceci était également le destin initialement conçu pour le bureau parisien par les réformateurs de l'Institut suédois.

5. Protokoll I970 : 2, bilaga I : "Varsel om utredning PM" från Utbildningsdepartementet, Kulturenheten, 3I.3.1970. RA, SI2, Serie Ö2 (vol. 4) : 6-7.

6. Sauf autre mention, toutes les traductions sont les miennes.

7. La loi du 4 août I962, dite loi Malraux, du nom du ministre de la culture André Malraux, vise à compléter la législation sur la protection du patrimoine historique et esthétique français en facilitant la restauration immobilière pour conserver l'intégrité des quartiers et monuments historiques.

8. Utbildningsdepartementet : Stencil I970 : I 5. PM. "Svenska kulturhuset i Paris, verksamhet och organisation ", mémorandum du 8.7.I970. RA, SI2, Serie FI3 (vol. 3) : 2 .

9. Petri \& Lundberg au gouvernement suédois "Styrelsen för Institut Tessin i Paris ang. institutets lokalfråga”, Io/3-65. Bilaga 2, Hans Nilsson-Ehle "Synspunkter på den framtida verksamheten vid Institut Tessin i Paris", 5.3.1965. Bilaga 3, Sten Karling, "Några synspunkter på Institut Tessin och dess uppgifter", 6.3.1965. RA, SI2, Serie Ö2, Vol. 3 .

Io. Sur l'usage du concept de diplomatie publique dans le contexte de l'Institut suédois, voir Glover (20 I I : I 83).

II. Le Ministère des Affaires ecclésiastiques est à partir du 3I janvier I967 devenu le Ministère de l'Éducation.

I2. Pour une étude récente de la formation de la catégorie de politique culturelle en Suède, voir M. Klockar Linder (20I4), Kulturpolitik. Formeringen av 
en modern kategori. Uppsala Studies in History of Ideas 45. Uppsala : Acta Universitatis Upsaliensis.

I3. Voir par exemple Frenander (I998 : I 58 -I 59).

I4. Ce travail aboutira à une publication sur l'image de la Suède à l'étranger, à savoir G. Stridsberg (éd.) (1958), Sverige-bilden i utlandet : Utdrag ur Svenska institutets klippbok I95I-57. Stockholm : Svenska Institutet.

I 5. Utbildningsdepartementet : Stencil I970 : I 5. PM. "Svenska kulturhuset i Paris, verksamhet och organisation ", mémorandum du 8.7.I970. RA, SI2, Serie FI3 (vol. 3) : IO-I I.

I6. Utbildningsdepartementet : Stencil I970 :I 5. PM. "Svenska kulturhuset i Paris, verksamhet och organisation", mémorandum du 8.7.I970. RA, SI2, Serie FI3 (vol. 3) : 22 .

I7. Hildeman, "PM angående kulturverksamheten i det svenska kulturhuset i Paris”, mémorandum du I6.Io.I968. RA, SI2, Serie FI3 (vol. 3) : I.

\section{I8. Ibid.}

I9. Pour une analyse plus approfondie de Hildeman et de ses perspectives sur la nature du travail de l'Institut suédois, voir Glover (20II), en particulier "Public relations and the public's relations of the nation ", I3 $38-\mathrm{I} 55$.

20. Utbildningsdepartementet : Stencil I970:I 5. PM. "Svenska kulturhuset i Paris, verksamhet och organisation", mémorandum du 8.7.1970. RA SI2, Serie FI3 (vol. 3) : 23-24.

2I. Grate, "Rapport över verksamheten vid Pariskontoret I.I-3I.3 I97I", 2. CCS, Serie BI (vol. 2).

22. Ibid.

\section{Références}

\section{Sources non publiées :}

Riksarkivet (RA) (Archives nationales de Suède) (RA),

Svenska institutet 2 (SI2), Serie $\mathrm{F}_{3}$, Vol. 3 ; Serie Ö2, Vol. $3-4$.

Svenska institutet i Paris (CCS) (Institut suédois à Paris), Serie Bı, Vol. 2.

\section{Sources publiées}

Martinet, G. (I969), «Vérités sur le ‘modèle suédois’ ». Le Nouvel Observateur, 30 juin. 
Servan-Schreiber, J.-J. (I967), Le défi américain. Paris : Denoël.

SIUP I968 (Sverige i utländsk press/La Suède dans la presse étrangère).

SIUP I969 (Sverige i utländsk press/La Suède dans la presse étrangère).

Stridsberg, G. (éd.) (I958), Sverige-bilden i utlandet: Utdrag ur Svenska institutets klippbok I95I-57. Stockholm : Svenska Institutet.

\section{Bibliographie}

Cull, N. J. (2008), The Cold War and the United States Information Agency: American propaganda and public diplomacy, I945-1989. Cambridge : Cambridge University Press.

Faramond, G. de (2000), La Suède et la France. Stockholm : Institut suédois.

Frenander, A. (I998), Debattens vågor: Om politisk-ideologiska frågor i efterkrigstidens svenska kulturdebatt. Arachne I3. Göteborg : Institutionen för idé- och lärdomshistoria.

Glover, N. (2009), "Imaging Community : Sweden in 'cultural propaganda' then and now ». Scandinavian Journal of History, vol. 34, n. 3: 246-263.

- (20II). National relations. Public diplomacy, national identity and the Swedish Institute, 1945-1970. Lund : Nordic Academic Press.

Hellenes, A. M. (20I 5, à paraître), "Institut suédois et images de la Suède à Paris dans l'après-guerre ". Revue d'histoire nordique I9.

Hildeman, P.-A. (I995), Upplysningsvis : Svenska institutet I945-I995. Stockholm : Almqvist \& Wiksell.

Paschalidis, G. (2009), "Exporting national culture: histories of Cultural Institutes abroad ». International Journal of Cultural Policy 3 (vol. I 5 ) : 275-289.

Stråth, B. (2006), « La construction d'un modèle nordique : pressions externes et compromis interne ". Revue internationale de politique comparée 3 (vol. I3) : 39I-4II.

Svenstedt, C. H. (I993), "La petite maison dans le Marais », in Battail, M. \& J.-F. (éd.), Une Amitié millénaire. Les relations entre la France et la Suède à travers les âges. Paris : Beauchesne : 473-492.

Whitling, F. (20II), "Relative Influence: Scholars, institutions and academic diplomacy in post-war Rome. The case of the German libraries (I943-53) ». The International History Review 4 (vol. 33) : 645-668. 
Östberg, K. \& Andersson, J. (éd.) (2013), Sveriges historia 1965-20I2. Stockholm : Norstedts.

Åkerlund, A. (20II), "Kulturtransfer och kulturpolitik ", in Åkerlund, A. (éd.), Kulturtransfer och kulturpolitik. Uppsala : Swedish Science Press (Opuscula historica Upsaliensia) : 5-16. 